\title{
Neuroendocrine rectal tumour as a rare aetiology of adult recto-sigmoidal intussusception: case presentation and literature review
}

\author{
Address for correspondence: \\ Bogdan Cristea \\ "Carol Davila" University of Medicine \\ and Pharmacy of Bucharest \\ 8 Eroilor Sanitari Blvd., \\ RO-050474 Bucharest, Rumania \\ e-mail: bogdanc_73@yahoo.com
}

Oncology in Clinical Practice 2018, Vol. 14, No. 5, 282-285 DOI: $10.5603 / \mathrm{OCP} .2018 .0036$ Copyright $(\odot) 2018$ Via Medica ISSN 2450-1654

\begin{abstract}
Intestinal intussusception is a frequent paediatric pathology, but adult cases are rare and do not have a specific presentation. In most cases adult intestinal intussusception, regardless of its localisation, is caused by an endoluminal tumour, which can be of multiple pathological types. We report a sigmoido-rectal intussusception in an adult in whom an adenoneuroendocrine tumour was identified as the lead point. This case is unique for the following reasons: (1) neuroendocrine tumours rarely involve the sigmoido-rectal segment of the gut, (2) clinical presentation with a recto-sigmoidal intussusception presented a diagnostic challenge.
\end{abstract} Key words: rectal intussusception, intussusception, neuroendocrine tumour, surgery

Oncol Clin Pract 2018; 14, 5: 282-285

\section{Introduction}

Adult intussusception is a rare aetiology for intestinal obstruction occurring in 1 to $5 \%$ of the total mechanical bowel obstructions [1]. Zubaidi et al. [2] reported that $86 \%$ of the adult intussusception had an organic lesion and $33 \%$ of those involving the colon were malignant. Plain radiography has low specificity and the diagnosis is made with a CT (computed tomography) scan which has an accuracy of $98 \%$ [3] and revealed a tumour in $72 \%$ of cases [3]. The pathognomonic finding on CT is the "target sign" (Figure 1). Surgical exploration is the traditional management because the lead point of the intussusception is usually malignant [4]. There are no reports in the literature of a neuroendocrine tumour causing recto-sigmoidal intussusception.

The annual incidence of all neuroendocrine tumours in the United States is estimated at 3.65 per 100,000 population [5]. Well differentiated neuroendocrine tumours have a $67 \%$ five-year survival rate [6], while the poorly differentiated neuroendocrine tumours have a much lower survival rate (34 months for those with localised disease and five months for those with metastatic disease [7]). They can be secreting tumours (gastrinoma, somatostatinoma, insulinoma, VIPoma) or non-secreting tumours. Although functionality may impact prognosis (e.g. insulinomas are generally indolent tumours), the biological behaviour of most functioning neuroendocrine tumours is defined by the grade and stage of the tumour. The treatment of choice for non-metastatic tumours is surgery, which is rarely curative by itself, and so adjuvant chemotherapy is also employed [8].

\section{Case presentation}

History

We present the case of a 53-year-old man, vegetarian, who was evaluated for anus prolapsing tumour. He reported $15-\mathrm{kg}$ weight loss (18\% body weight loss) over the prior year and low quantitative diarrhoea for 


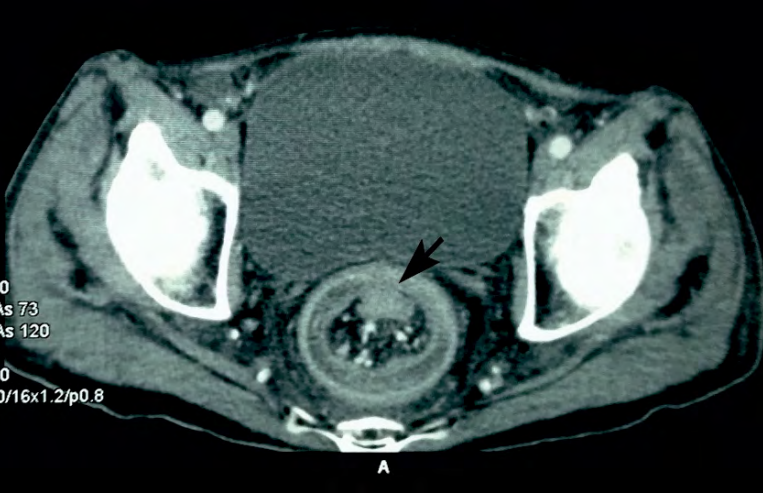

Figure 1. Computer tomography image of our patient showing the target sign of intussusception. It also shows the tumour inside the rectal lumen (black arrow)

the prior nine months. His medical history included insulin-treated type 2 diabetes mellitus. On physical examination, the patient looked cachectic and pale but had no abdominal complains. On rectal examination, the anal sphincter muscle was hypotonic with a $3 \mathrm{~cm}$ pediculate rectal tumour prolapsing through the anus and successive rectal intussusception. There was no associated local pain or bleeding. The rest of his physical examination was non-contributory.

\section{Laboratory and Imagistic investigations}

Blood analyses were only remarkable for mild hypochromic microcytic anaemia, mild hypokalaemia, and hyperglycaemia. Abdominal and thoracic plain radiographs were normal, as was an abdominal ultrasound. A computed tomography examination indicated a rectal tumour with successive intussusception (Figure 1). The intussusception was not complicated by intestinal obstruction or ischaemia, and there were no signs of local invasion or distant metastasis. A colonoscopy was performed, which demonstrated the tumour to be $25 \mathrm{~cm}$ from the anal verge (sigmoidal tumour). The biopsy of this tumour was reported as squamous cell carcinoma.

\section{Treatment}

Considering the deconditioned status of the patient, a transanal local excision with full thickness rectal wall resection was performed, followed by rectal suture. A manual reduction of the intussusception was performed. The immediate postoperative course was favourable, but on the second postoperative day the intussusception relapsed upon defecation. A colonoscopy-aided reduction was attempted during the eighth postoperative day (delayed by the full thickness rectal suture) without success. An open recto-sigmoidian resection (Hartmann procedure) was performed (Figure 2) with resection of the intussuscepted segment

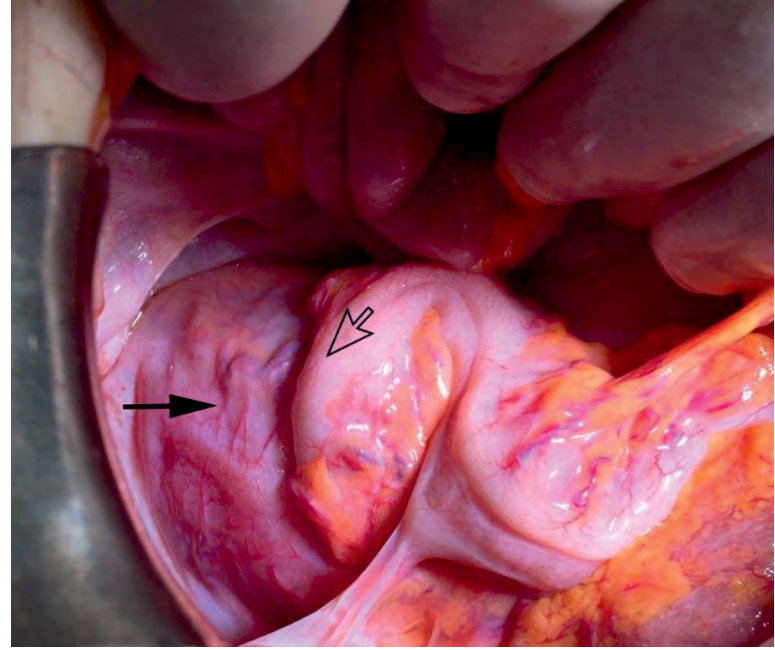

Figure 2. Intraoperative findings: Distal sigmoid colon (hollow arrow) invaginated through the upper rectum (full arrow)

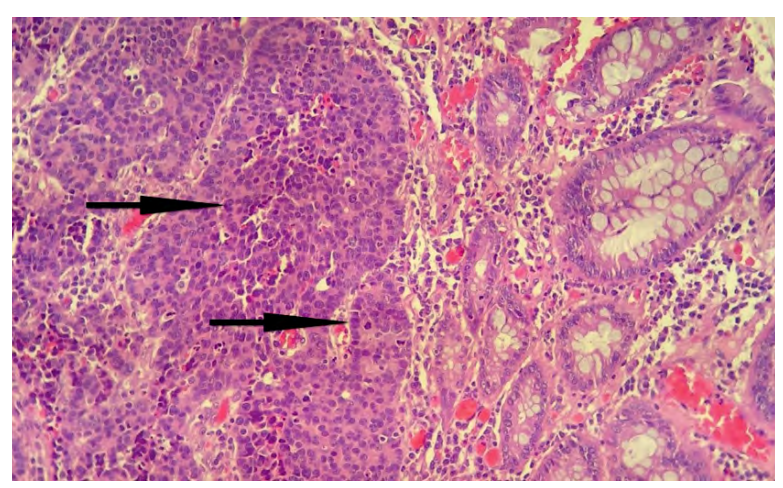

Figure 3. Haematoxylin-Eosin staining ( $\times 200$ magnification) showing a poorly-differentiated neuroendocrine tumour (black arrow indicate tumour cell)

and left iliac colostomy. The postoperative period was uneventful.

The final pathology report showed a $6 / 4 \mathrm{~cm}$ poorly-differentiated neuroendocrine tumour with large cell pattern (Figure 3), frequent mitosis (> 20/10 high-power field), with tumour necrosis, no lympho-vascular invasion, a pT2 depth invasion, and no lymph nodes invaded (out of 17 examined). The immunohistochemistry test showed a positive synaptophysin (Figure 4), negative chromogranin, CD56 (neural cell adhesion molecule) positive in more than $50 \%$ of cells, CDX2 (caudal-type homeobox transcription factor 2) positive, TTF1 (thyroid transcription factor) and p53 negative, and Ki-67 positive in $35-40 \%$ of cells.

A diagnosis of poorly-differentiated neuroendocrine carcinoma was made, and the patient underwent six cycles of carboplatin and etoposide chemotherapy. At the six-month follow-up there was no evidence of tumour recurrence or distant metastasis. 


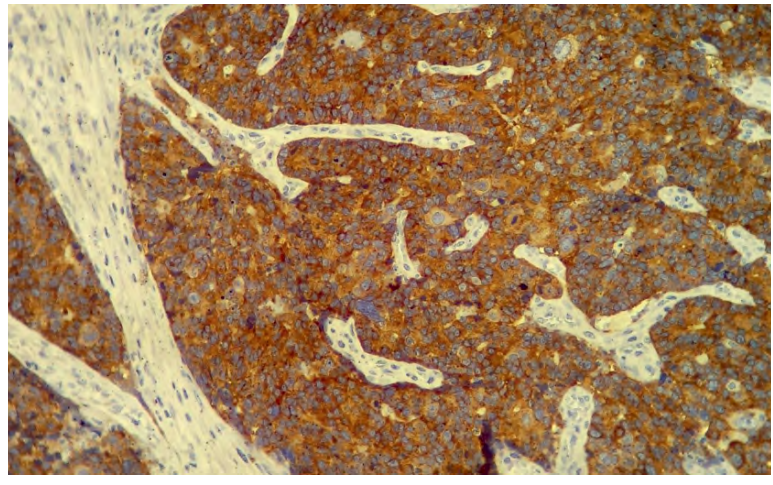

Figure 4. Synaptophysin staining ( $\times 200$ magnification) supporting the diagnosis of neuroendocrine tumour

\section{Discussion}

Lindor et al. reported that most patients with intestinal intussusception present in the ambulatory setting (69\%), and the most frequent complaint is abdominal pain (62\%) [3]. Our patient presented with a rectal prolapsing tumour without bowel obstruction. Malignancy was suspected because of the associated weight loss, but also because of clinical presentation with recto-sigmoidal intussusception. Although rare, recto-sigmoidal intussusception occurs most often on an organic lead point, of which malignant causes are frequent. Eisenet et al. found that $80 \%$ of the colonic intussusceptions were malignant [9]. Conversely, Azaret et al. reported that $43 \%$ of the colonic neuroendocrine tumours were malignant and $57 \%$ benign [10]. Our patient had a poorly differentiated neuroendocrine tumour, a tumour that has not previously been reported to present with intestinal intussusception.

Neuroendocrine tumours are rare and can occur in the gastrointestinal tract and pulmonary system. Gastroeneteropancreatic neuroendocrine tumours have an incidence of 5.25/100,000/year, of which a very small subset are localised in the rectum (incidence of rectal neuroendocrine tumours is $0.86 / 100,000 /$ year [11]). Neuroendocrine tumours have the highest incidence in the fifth decade of life and a slight male predominance [7]. Functioning neuroendocrine tumours can secrete insulin, gastrin, VIP, or somatostatin, which may or may not cause an associated clinical syndrome. Identification of hormone staining on immunohistochemical analysis does not prove the functionality of the tumour [11], and functionality is not a predictor of prognosis. Our patient presented with diarrhoea, hypokalaemia, and hyperglycaemia, which are compatible with Verner-Morrison syndrome (excessive VIP hormone). We believe our patient's diarrhoea was due to anal incontinency (hypotonic sphincter) because it was not secretory-watery type and was low quantitative, while his hyperglycaemia was due to uncontrolled diabetes. Furthermore, most VIPomas are localised in the pancreas (more than 95\%) [12]. Nonetheless, immunohistochemical staining of the tumour did not identify any hormone in the cells. Most rectal neuroendocrine tumours are nonfunctioning; PP, somatostatin, and PYY are the most common products of the secretory subtypes [11].

Staging and differentiation are good parameters of prognosis. According to TNM seventh edition and WHO grading from 2010, our patient had a pT-2N0M0 G3 poorly-differentiated neuroendocrine tumour. Distant metastasis are present in $67 \%$ of patients at the time of diagnosis of high-grade neuroendocrine carcinomas [13].

North American Neuroendocrine Society (NANETS) [14] group recommends surgical resection followed by chemotherapy for most T1-T2 tumour stages, even though prognosis is poor for these high-grade neuroendocrine carcinomas ( 38 months mean survival) [15] and even worse for large bowel tumours (only 15\% are alive at one year from diagnosis [16]).

\section{Conclusions}

We presented a unique case of a 53-year-old male patient who presented with asymptomatic rectal intussusception due to a neuroendocrine tumour. Rectal intussusception is a rare condition in adults and has no specific presenting symptoms. The clinician must have a high index of suspicion in such cases and must remember that ultrasonography and plain abdominal $\mathrm{X}$-ray rarely diagnose the pathology, but may show the associated obstruction if present. Computed tomography imaging has a high accuracy for the diagnosis and can also show the lead point of the intussusception, which in large bowel is frequently a malignancy. Neuroendocrine tumours of the rectum are rare and usually non-secretory. The treatment in early stages is surgery combined with chemotherapy. High-grade poorly-differentiated neuroendocrine tumours have a poor prognosis.

Conflict of interest: The authors report no conflicts of interest

\section{References}

1. Marinis A, Yiallourou A, Samanides L, et al. Intussusception of the bowel in adults: a review. World J Gastroenterol. 2009; 15(4): 407-411, doi: 10.3748/wjg.15.407, indexed in Pubmed: 19152443.

2. Zubaidi A, Al-Saif F, Silverman R. Adult intussusception: a retrospective review. Dis Colon Rectum. 2006; 49(10): 1546-1551, doi: 10.1007/s10350-006-0664-5, indexed in Pubmed: 16990978.

3. Lindor RA, Bellolio MF, Sadosty AT, et al. Adult intussusception: presentation, management, and outcomes of 148 patients. J Emerg Med. 2012; 43(1): 1-6, doi: 10.1016/j.jemermed.2011.05.098, indexed in Pubmed: 22244289 
4. Erkan N, Haciyanli M, Yildirim M, et al. Intussusception in adults: an unusual and challenging condition for surgeons. Int $\mathrm{J}$ Colorectal Dis. 2005; 20(5): 452-456, doi: 10.1007/s00384-004-0713-2, indexed in Pubmed: 15759123.

5. Lawrence B, Gustafsson BI, Chan A, et al. The epidemiology o gastroenteropancreatic neuroendocrine tumors. Endocrinol Metab Clin North Am. 2011; 40(1): 1-18, vii, doi: 10.1016/i.ecl.2010.12.005, indexed in Pubmed: 21349409.

6. Ramage JK, Davies AHG, Ardill J, et al. UKNETwork for Neuroendocrine Tumours. Guidelines for the management of gastroenteropancreatic neuroendocrine (including carcinoid) tumours. Gut. 2005; 54 Suppl 4 iv1-i16, doi: 10.1136/gut.2004.053314, indexed in Pubmed: 15888809.

7. Yao JC, Hassan M, Phan A, et al. One hundred years after "carcinoid": epidemiology of and prognostic factors for neuroendocrine tumors in 35,825 cases in the United States. J Clin Oncol. 2008; 26(18): 3063-3072, doi: 10.1200/JCO.2007.15.4377, indexed in Pubmed: 18565894.

8. Brenner B, Tang LH, Klimstra DS, et al. Small-cell carcinomas of the gastrointestinal tract: a review. J Clin Oncol. 2004; 22(13): 2730-2739, doi: 10.1200/JCO.2004.09.075, indexed in Pubmed: 15226341

9. Eisen LK, Cunningham JD, Aufses AH. Intussusception in adults: institutional review. J Am Coll Surg. 1999; 188(4): 390-395, indexed in Pubmed: 10195723.

10. Azar T, Berger DL. Adult intussusception. Ann Surg. 1997; 226(2): 134-138, doi: 10.1097/00000658-199708000-00003, indexed in Pubmed: 9296505.
11. Oberg K, Knigge U, Kwekkeboom D, et al. Neuroendocrine gastro-entero-pancreatic tumors: ESMO Clinical Practice Guidelines for diagnosis, treatment and follow-up. Annals of Oncology. 2012; 23(suppl 7): vii124-vii130, doi: 10.1093/annonc/mds295.

12. Friesen SR. Update on the diagnosis and treatment of rare neuroendocrine tumors. Surg Clin North Am. 1987; 67(2): 379-393, doi: 10.1016/s0039-6109(16)44190-3, indexed in Pubmed: 3031836

13. Smith JD, Reidy DL, Goodman KA, et al. A retrospective review of 126 high-grade neuroendocrine carcinomas of the colon and rectum. Ann Surg Oncol. 2014; 21 (9): 2956-2962, doi: 10.1245/s10434-014-3725-3, indexed in Pubmed: 24763982

14. Strosberg JR, Coppola D, Klimstra DS, et al. North American Neuroendocrine Tumor Society (NANETS). The NANETS consensus guidelines for the diagnosis and management of poorly differentiated (high-grade) extrapulmonary neuroendocrine carcinomas. Pancreas. 2010; 39(6): 799-800, doi: 10.1097/MPA.0b013e3181ebb56f, indexed in Pubmed: 20664477

15. SEER ${ }^{\star}$ Stat Database: Incidence - SEER 9 RegsResearch Data, November 2011 submission (1973-2010). Bethesda, MD: National Cancer Institute, Cancer StatisticsBranch; 2013. www.seer cancer.gov.

16. Hung SS. Small cell carcinoma of the colon. A case report and literature review. J Clin Gastroenterol. 1989; 11(3): 335-339, doi: 10.1097/00004836-198906000-00019, indexed in Pubmed: 2546989 . 